\title{
Evaluación de las concentraciones séricas de procalcitonina como indicador de gravedad y mortalidad en sepsis abdominal por peritonitis secundaria
}

\author{
Evaluation of the serum procalcitonin level as an indicator of severity and mortality in \\ abdominal sepsis due to secondary peritonitis
}

\author{
Ansony R. Godínez-Vidal'", Verónica Rojas-Hernández¹, Paola J. Montero-García ${ }^{2}$, \\ Antonio R. Martínez-Martínez ${ }^{2}$, Julio C. Zavala-Castillo ${ }^{2}$ y Noé I. Gracida-Mancilla ${ }^{2}$ \\ ${ }^{1}$ Departamento de Cirugía General; ${ }^{2}$ Clinica de Sepsis Abdominal, Departamento de Cirugía General. Hospital General de México Dr. Eduardo \\ Liceaga, Ciudad de México, México
}

\begin{abstract}
Resumen
Introducción: La procalcitonina es un biomarcador de sepsis, cuyas concentraciones aumentan cuando alguna endotoxina entra en el torrente sanguíneo. Se emplea, entre otras cosas, para discriminar la etiología de las infecciones, escalar/desescalar antibióticos, aumentar o disminuir el espectro antibiotico, y predecir la mortalidad. Objetivo: Determinar si los valores de procalcitonina se relacionan con la gravedad y la mortalidad de los pacientes con sepsis abdominal. Método: Estudio retrospectivo, descriptivo y transversal de pacientes con diagnóstico de sepsis abdominal, de abril de 2016 a febrero de 2017. En todos los casos se determinó la gravedad mediante las escalas APACHE II, SOFA, Mannheim y CONUT, así como la mortalidad. Se dividió la muestra en pacientes con procalcitonina $>10.1$ y < 10. Resultados: Se incluyeron 99 casos (41 mujeres y 58 hombres). El principal órgano causante de sepsis abdominal fue el apéndice (56\%). La media de procalcitonina para la muestra se situó en 7.94 (desviación estándar: \pm 13.76$)$. Los hallazgos, sometidos a verificación estadística mediante la prueba $U$ de Mann-Whitney, mostraron una relación estadísticamente significativa en los casos con procalcitonina 10.1, puntajes de Mannheim > 26 puntos $(p=0.003)$, CONUT $>6$ puntos $(p=0.027)$ y presencia de fallas orgánicas $(p=0.001)$; no hubo relación significativa entre los valores de procalcitonina y los puntajes de las escalas APACHE II y SOFA, ni con la mortalidad. Conclusión: La gravedad medida por la procalcitonina se relaciona con la gravedad determinada mediante los índices de Mannheim y CONUT, y con el desarrollo de fallas orgánicas.
\end{abstract}

PALABRAS CLAVE: Gravedad. Mortalidad. Procalcitonina. Sepsis.

\begin{abstract}
Background: Procalcitonin is a biomarker of sepsis, whose concentrations increase when some endotoxin enters the bloodstream. It is used, among other things, to discriminate the etiology of infections, increase or decrease the antibiotic spectrum, and predict mortality. Objective: To determine the utility of the serum level of procalcitonin as a predictor of severity and mortality. Method: Retrospective, descriptive, cross-sectional study of patients diagnosed with abdominal sepsis during the period from April 2016 to February 2017. In all cases the severity was determined by APACHE II, SOFA, Mannheim and CONUT, and mortality. The sample was divided into those with procalcitonin > 10.1 and < 10. Results: We included 99 cases (41 female and 58 male). The main organ causing abdominal sepsis was the appendix $56 \%$. The mean of procalcitonin for the sample was 7.94 (standard deviation: \pm 13.76). The findings, subjected to statistical verification by means of the Mann-Whitney $U$ test, showed statistical significance among the cases with procalcitonin 10.1, with the Mannheim scores > 26 points $(p=0.003)$, CONUT >
\end{abstract}

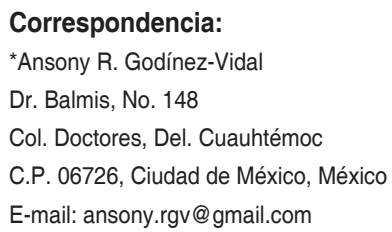

Fecha de recepción: 25-03-2018

Fecha de aceptación: 05-05-2018

DOI: 10.24875/CIRU.18000301
Cir Cir. 2019;87:1-259

Contents available at PubMed www.cirugiaycirujanos.com 
6 points $(p=0.027)$ and presence of organic faults $(p=0.001)$, but not with APACHE, SOFA and mortality. Conclusions: Procalcitonin is related to the severity determined by the Mannheim index, CONUT and the development of organic faults.

KEY WORDS: Mortality. Procalcitonin. Sepsis. Severity.

\section{Introducción}

La procalcitonina (PCT) es una prohormona de origen proteico, parecida a la calcitonina, de la cual es el péptido precursor. Las concentraciones séricas normales de PCT en los humanos sanos son $<0.1 \mathrm{ng} / \mathrm{ml}^{1}$. En condiciones normales, es producida y secretada por las células $C$ en la glándula tiroidea. También es secretada por células neuroendocrinas del pulmón y el intestino; estas dos últimas fuentes de PCT proporcionan su verdadera utilidad clínica, ya que aumentan su producción en respuesta a un estímulo proinflamatorio. Los valores de PCT pueden aumentar a más de 400 veces la línea base (> $4 \mathrm{ng} / \mathrm{ml}$ ) rápidamente cuando una endotoxina entra en el torrente sanguíneo ${ }^{2}$.

Se han sugerido que el valor de la PCT puede determinar el riesgo y la gravedad de una infección: con PCT $<0,5 \mathrm{ng} / \mathrm{ml}$, no hay riesgo de infección; con PCT de 0,5$2 \mathrm{ng} / \mathrm{ml}$, el riesgo de infección es moderado; con PCT de $2-10 \mathrm{ng} / \mathrm{ml}$, hay alto riesgo de progresión hacia una infección sistémica grave; y con PCT > $10 \mathrm{ng} / \mathrm{ml}$ existe una alta probabilidad de sepsis grave o shock séptico ${ }^{3}$.

Son numerosas las indicaciones para el uso de la PCT como marcador:

- Diagnóstico de infección bacteriana en presencia de síndrome de respuesta inflamatoria sistémica.

- Monitorización de la terapia con antibióticos y evolución de la infección bacteriana.

- Diagnóstico diferencial de enfermedades inflamatorias y fiebre de origen desconocido.

- Predecir la mortalidad.

En la Conferencia Internacional de Definiciones de Sepsis del año 2001 se estableció que lo más importante es el diagnóstico en las etapas iniciales de la respuesta inflamatoria a la infección, y cuantificar su gravedad, por lo que es prioritario identificar los síntomas y los signos que sugieran la sospecha de sepsis ${ }^{4,5}$.

Los síntomas y los signos específicos de sepsis pueden no aparecer inicialmente, y el diagnóstico microbiológico puede tardar incluso días. Sin embargo, en caso de no iniciar un tratamiento enérgico (fluidoterapia, fármacos vasoactivos y antibioticoterapia empírica) precozmente, el riesgo de desarrollar fallo multiorgánico y de que se produzca el fallecimiento del paciente es alto ${ }^{6,7}$.
El valor diagnóstico de los marcadores de inflamación podría diferenciar procesos infecciosos de los que no lo son, y predecir la gravedad de un proceso patológico o enfermedad, lo que permitiría iniciar un plan terapéutico adecuado y medir su respuesta ${ }^{8-11}$.

El objetivo de la presente investigación es determinar la utilidad de la determinación de las concentraciones séricas de PCT como predictor de gravedad y de mortalidad en pacientes con sepsis abdominal.

\section{Método}

Se realizó un estudio retrospectivo, descriptivo y transversal de pacientes con diagnóstico de sepsis abdominal por peritonitis secundaria atendidos entre abril de 2016 y febrero de 2017. En todos los casos se determinó la gravedad de la sepsis abdominal mediante dos escalas fisiológicas Acute Physiology And Chronic Health Evaluation II y Acute Organ System Failure (APACHE II y SOFA), un índice quirúrgico (Mannheim) y un índice nutricional de Control Nutricional (CONUT), y mediante el desarrollo de fallas orgánicas y la mortalidad. En todos los pacientes se determinó la PCT. Se dividió la muestra en dos grupos: pacientes con PCT > 10.1 y pacientes con PCT < 10; se empleó este punto de discriminación porque en la literatura se ha reportado que con esta cifra existe una alta probabilidad de sepsis grave o shock séptico. Para las escalas e índices de gravedad se dividieron los casos en leves (APACHE II $<14$, SOFA $<3$, Mannheim $<25$ puntos y CONUT $<5$ ) y graves (APACHE II > 15, SOFA > 4, Mannheim > 26 y CONUT $>6$ ) (Tabla 1). Se compararon los grupos empleando tablas de $2 \times 2$ y se evaluaron las frecuencias mediante la prueba de ji al cuadrado. Para determinar la asociación entre las concentraciones séricas de PCT y las escalas de gravedad (APACHE II, SOFA, Mannheim y CONUT), el desarrollo de fallas orgánicas y la mortalidad, se empleó la prueba $U$ de Mann-Whitney como estadístico de prueba de hipótesis, y se estableció como significativo un valor de $p<0.05$.

\section{Resultados}

Se incluyeron 99 casos (41 mujeres y 58 hombres), con una media de edad de 43.4 años (desviación 
Tabla 1. Clasificación en casos leves y graves

\begin{tabular}{lcc}
\hline Indicador de gravedad & Graves & Leves \\
\hline APACHE II & $>15$ & $<14$ \\
SOFA & $>4$ & $<3$ \\
Mannheim & $>26$ & $<25$ \\
CONUT & $>6$ & $<5$ \\
Fallas orgánicas & Con fallas & Sin fallas \\
Desenlace & Fallecido & Vivo \\
\hline
\end{tabular}

Tabla 2. Relación de la procalcitonina $>10.1 \mathrm{ng} / \mathrm{ml}$ con las escalas de gravedad

\begin{tabular}{lccccc}
\hline Variable & \multicolumn{2}{c}{ Gravedad por PCT } & p & OR \\
\cline { 2 - 3 } & $\begin{array}{c}\text { Graves } \\
\text { PCT }>10.1 \mathrm{ng} / \mathrm{ml} \\
\text { Media (DE) }\end{array}$ & $\begin{array}{c}\text { Leves } \\
\text { PCT }<10 \mathrm{ng} / \mathrm{ml} \\
\text { Media (DE) }\end{array}$ & & \\
\hline APACHE II & $12(6.1)$ & $7.9(5.3)$ & 0.225 & - \\
SOFA & $4.3(1.5)$ & $2.8(1.6)$ & 0.102 & - \\
Mannheim & $20.6(7.3)$ & $16.1(6.4)$ & 0.003 & 5.2 \\
CONUT & $7.6(3.3)$ & $5.1(3.3)$ & 0.027 & 3.1 \\
\hline
\end{tabular}

DE: desviación estándar; OR: odds ratio; PCT: procalcitonina.

Tabla 3. Relación de la procalcitonina > $10.1 \mathrm{ng} / \mathrm{ml}$ con la presencia de fallas orgánicas y la mortalidad

\begin{tabular}{|c|c|c|c|c|}
\hline \multirow[t]{2}{*}{ Variable } & \multicolumn{2}{|c|}{ Gravedad por PCT } & \multirow[t]{2}{*}{$p$} & \multirow[t]{2}{*}{ OR } \\
\hline & $\begin{array}{c}\text { Graves } \\
\text { PCT }>10.1 \mathrm{ng} / \mathrm{ml} \\
\text { Media }(\mathrm{DE})\end{array}$ & $\begin{array}{c}\text { Leves } \\
\text { PCT }<10 \mathrm{ng} / \mathrm{ml} \\
\text { Media (DE) }\end{array}$ & & \\
\hline Fallas orgánicas & $59 \%(13 / 22)$ & $20.7 \%(16 / 77)$ & 0.001 & 0.187 \\
\hline Fallecimiento & $13.6 \%(3 / 22)$ & $2.5 \%(2 / 77)$ & 0.071 & - \\
\hline
\end{tabular}

DE: desviación estándar; OR: odds ratio; PCT: procalcitonina.

estándar [DE]: \pm 18.5 ). Los órganos causantes de la sepsis abdominal fueron el apéndice (56\%), el intestino delgado (18\%), el colon (12\%), el estómago ( $2 \%)$, las vías biliares (4\%), el útero y sus anexos $(1 \%)$, el páncreas $(1 \%)$, el riñón (3\%) y otros $(2 \%)$ (Fig. 1). Fallecieron $5(5.1 \%)$ pacientes y sobrevivieron 94 (94.9\%). Las puntuaciones medias de gravedad para la muestra fueron APACHE II 8.6 (DE: \pm 5.5 ), SOFA 3.1 (DE: \pm 1.6 ), Mannheim 17.2 (DE: \pm 7.4 ) y CONUT 5.5 (DE: \pm 3.5 ). La medición de la PCT para la muestra se situó con una media de $7.94 \mathrm{ng} / \mathrm{ml}$ (DE: \pm 13.76). Al comparar la cifra de PCT contra las escalas de gravedad (Tabla 2), los hallazgos, sometidos a verificación estadística mediante la prueba $U$ de Mann-Whitney, mostraron significación estadística en los casos con PCT > 10.1, puntajes de Mannheim $>26$ puntos ( $p=0.003)$ (Fig. 2) y
CONUT $>6$ puntos $(p=0.027)$ (Fig. 3), pero no con APACHE (Fig. 4) ni con SOFA (Fig. 5).

Al comparar los valores de PCT con la presencia de fallas orgánicas y la mortalidad (Tabla 3) se halló significación estadística para PCT > 10.1 y presencia de fallas orgánicas $(p=0.001)$ (Fig. 6$)$, pero no con la mortalidad (Fig. 7).

\section{Discusión}

En este estudio se comparó la PCT sérica en pacientes con sepsis abdominal, y se contrastaron sus valores contra los puntajes de gravedad obtenidos mediante las escalas SOFA, APACHE, Mannheim y CONUT, y por ultimo con la presencia de falla orgánica y mortalidad.

En un metaanálisis del año 2007 que incluyó 18 estudios publicados entre abril de 1996 y noviembre de 2005, Tang, et al. ${ }^{12}$ concluyeron que la PCT no permite discriminar entre la sepsis y el síndrome de respuesta inflamatoria sistémica.

Otros investigadores han sugerido que los biomarcadores pueden ser más útiles para descartar la sepsis que para diagnosticarla. Pierrakos ${ }^{13}$, en una revisión de 178 biomarcadores diferentes que fueron evaluados en 3370 estudios, identificó tres con un alto valor predictivo negativo para descartar la sepsis: la PCT $(99 \% \text { con un valor de corte de } 0,2 \mathrm{ng} / \mathrm{ml})^{14}$, la forma de onda del tiempo parcial de tromboplastina $(96 \%)^{15}$ y los productos de degradación de la fibrina (100\% para sepsis por gramnegativos mediante Enzyme-Linked ImmunoSorbent Assay (ELISA)) $)^{16}$.

En un estudio multinacional, prospectivo y observacional, realizado en 2015 por Schuetz, et al. ${ }^{17}$, se evaluó el uso de la PCT y de otros dos biomarcadores como estratificación del riesgo. Se encontró que la PCT predecía fuertemente la muerte dentro de los 30 días siguientes (área bajo la curva $[A \cup C]$ ): 0,75), la admisión en la unidad de cuidados intensivos (AUC: 0,62 ) y la alta prioridad en el triaje inicial (AUC: 0,58 ). Los otros dos biomarcadores incluidos en este estudio fueron la proadrenomedulina y la copeptina, que también fueron buenos predictores de los tres resultados.

En nuestro estudio hubo una mortalidad del 5.1\%. Al analizar los datos, la PCT no mostró significación estadística para predecir la mortalidad, pero sí se correlacionó con la presencia de fallas orgánicas.

La medición de la PCT se situó con una media de $7.94 \mathrm{ng} / \mathrm{ml}$, aunque hubo una gran dispersión en los valores de la muestra. De acuerdo con lo reportado en la literatura, valores $>10 \mathrm{ng} / \mathrm{ml}$ son indicativos de 


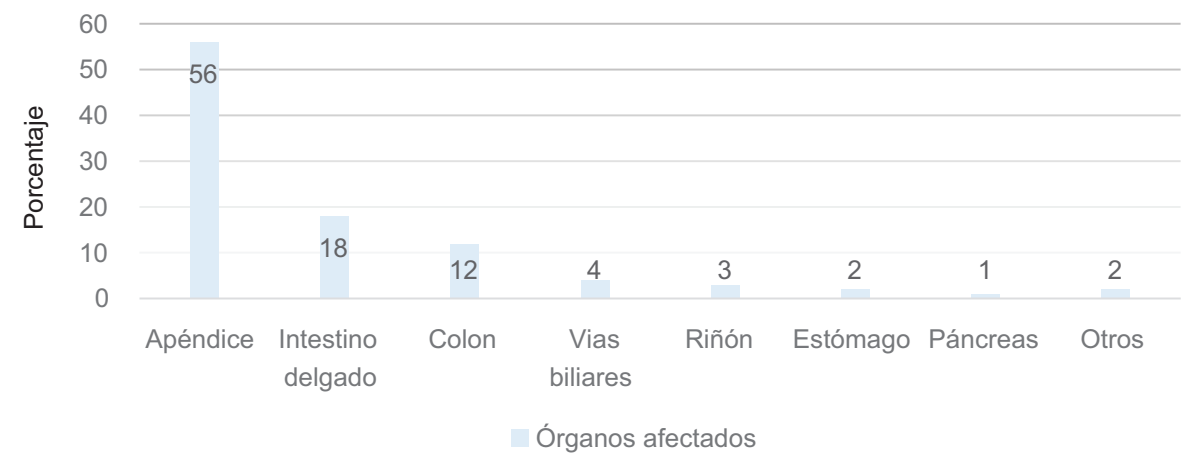

Figura 1. Órganos causantes de sepsis abdominal.

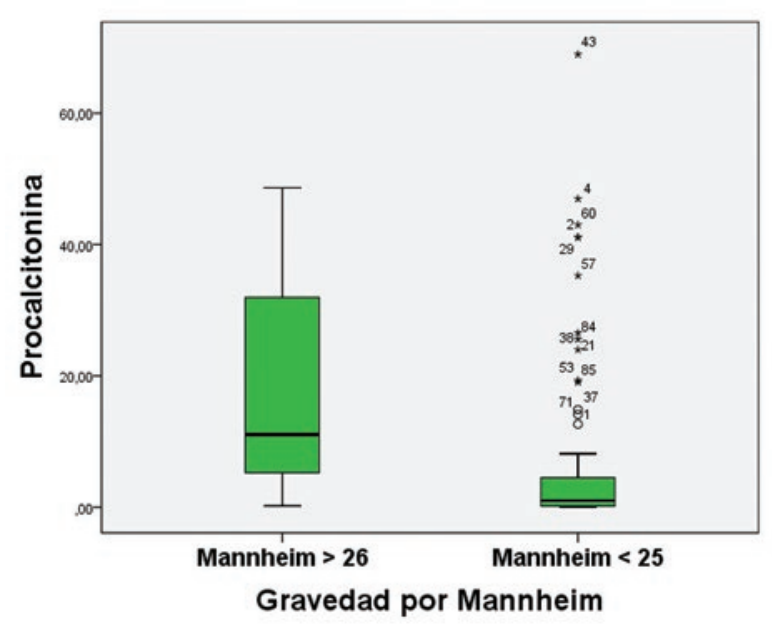

Figura 2. Relación entre los valores de procalcitonina y el índice Mannheim. En el gráfico se aprecia que a mayor valor de la procalcitonina, mayor puntaje del índice de Mannheim.

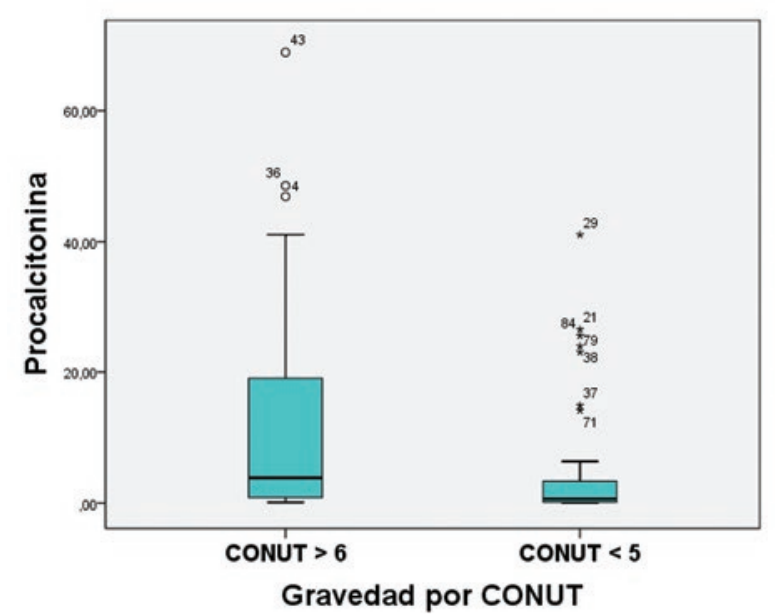

Figura 3. Relación entre los valores de procalcitonina y las puntuaciones en la escala CONUT. En el gráfico se aprecia que a mayor valor de procalcitonina, mayor calificación de CONUT.

alta probabilidad de sepsis grave o shock séptico ${ }^{3}$. Los valores de la PCT se correlacionaron de manera significativa con el puntaje de Mannheim y la escala

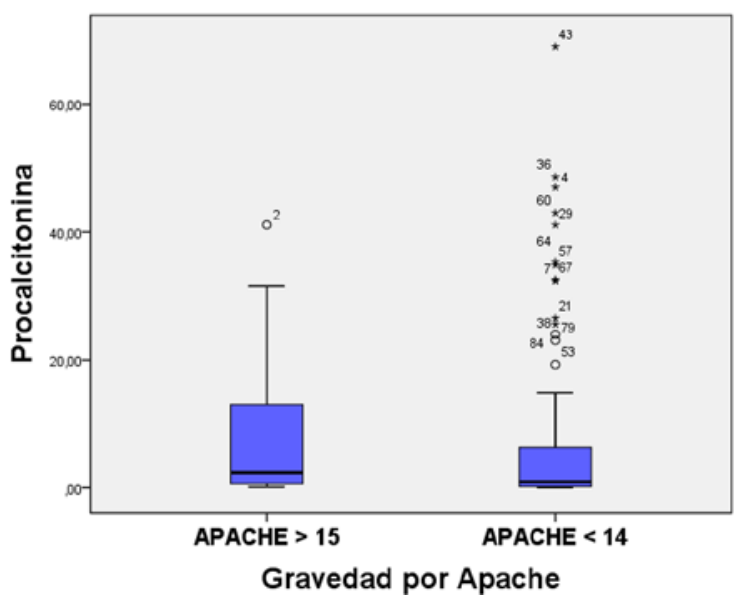

Figura 4. Relación entre los valores de procalcitonina y las puntuaciones en la escala APACHE. En el gráfico se aprecia que a mayor valor de la procalcitonina, mayor puntaje de APACHE, pero sin significación estadística.

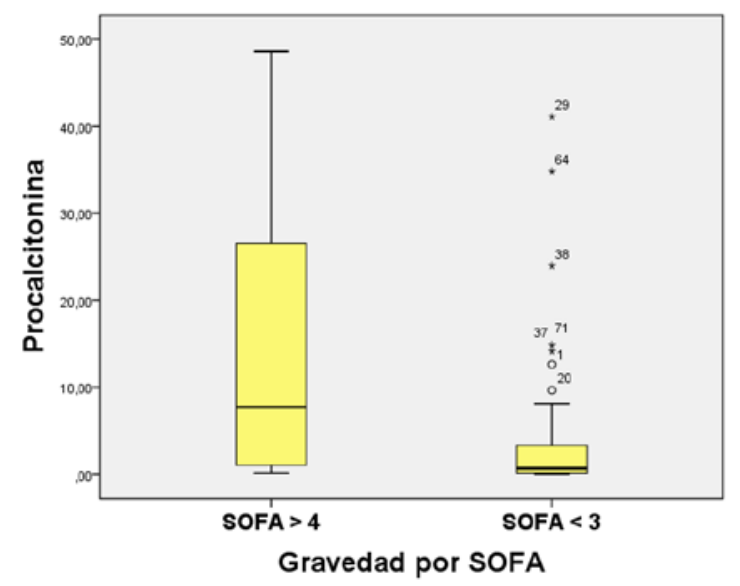

Figura 5. Relación entre los valores de la procalcitonina y las puntuaciones de la escala SOFA. En el gráfico se aprecia que a mayor valor de la procalcitonina, mayor puntaje de la escala SOFA, pero sin significación estadística.

CONUT para determinar la gravedad, pero no hubo significación estadística para las calificaciones de APACHE ni SOFA, ni para la mortalidad. 


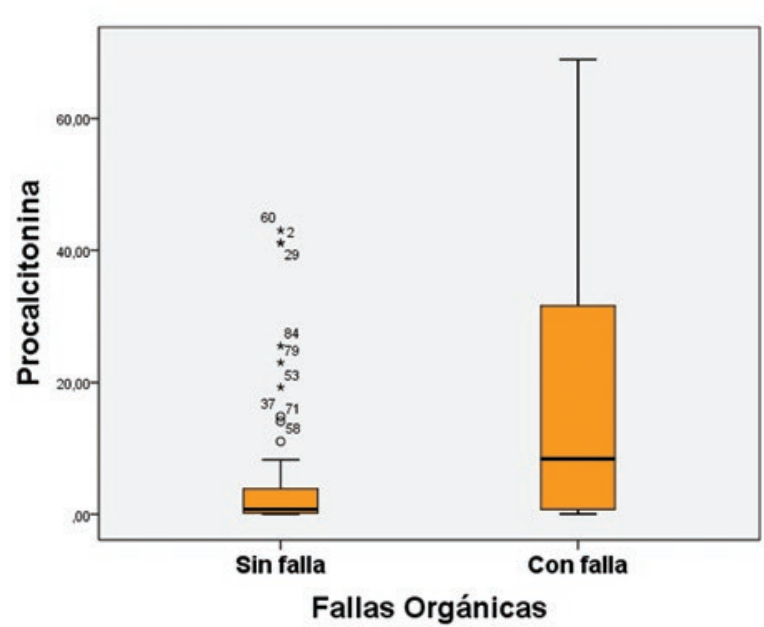

Figura 6. Relación entre los valores de procalcitonina y la presencia de fallas orgánicas. En el gráfico se aprecia que a mayor valor de la procalcitonina, mayor presencia de fallas orgánicas.

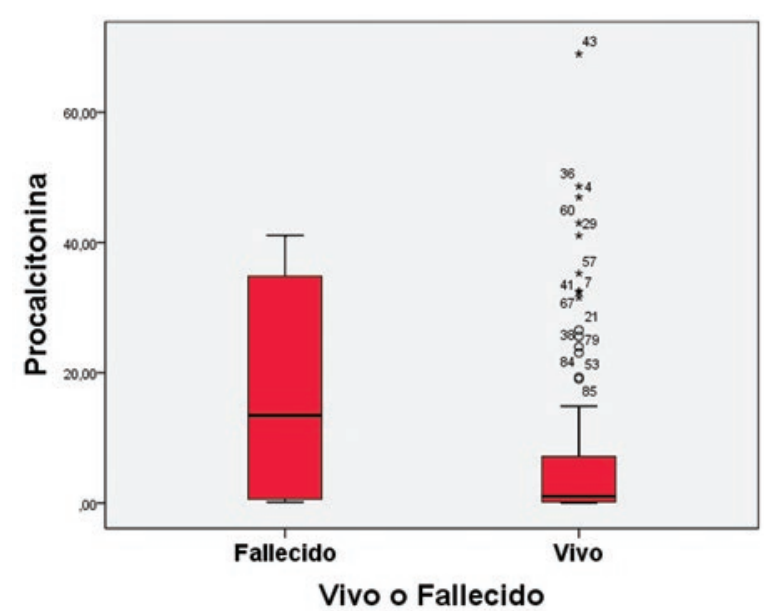

Figura 7. Relación entre los valores de procalcitonina y la mortalidad. En el gráfico se aprecia que a mayor valor de la procalcitonina, mayor mortalidad, pero sin significación estadística.

\section{Conclusión}

La PCT se relaciona con la gravedad determinada mediante las escalas de Mannheim y CONUT, y con el desarrollo de fallas orgánicas, pero no guarda relación con la gravedad determinada mediante APACHE II y SOFA. La PCT no es un buen predictor de mortalidad en la peritonitis. En nuestra muestra, la media de los valores de la PCT se sitúa en $7.9 \mathrm{ng} / \mathrm{ml}$.

\section{Financiamiento}

No se obtuvo ningún tipo de financiamiento por parte de alguna empresa.

\section{Conflicto de intereses}

No existe ningún conflicto de intereses.

\section{Responsabilidades éticas}

Protección de personas y animales. Los autores declaran que para esta investigación no se han realizado experimentos en seres humanos ni en animales.

Confidencialidad de los datos. Los autores declaran que han seguido los protocolos de su centro de trabajo sobre la publicación de datos de pacientes.

Derecho a la privacidad y consentimiento informado. Los autores declaran que en este artículo no aparecen datos de pacientes.

\section{Bibliografía}

1. Christ-Crains M, Müller B. Procalcitonin on the dusty way to the holy grail: a progress report. En: Vicent JL, editor. Yearbook of intensive care and emergency medicine. Berlín: Springer-Verlag; 2005. p. 461-76.

2. Dandona P, Nix D, Wilson MF, Aljada A, Love J, Assicot M, et al. Procalcitonin increase after endotoxin injection in normal subjects. J Clin Endocrinol Metab. 1994;79:1605-8.

3. Morgenthaler NG, Struck J, Fischer-Schulz C, Bergmann A. Sensitive immunoluminometric assay for the detection of pro-calcitonin (Department BRAHMS AG, Biotechnology Centre). Clin Chem. 2002;28:788-9.

4. Levy MM, Fink MP, Marshall JC, Abraham E, Angus D, Cook D, et al. 2001 SCCM/ESICM/ACCP/ATS/SIS International Sepsis Definitions Conference. Intensive Care Med. 2003;29:530-8.

5. León Gil C, García-Castrillo Riesgo L, Moya Mir M, Artigas Raventós A Borges SM, Candewl González FJ, et al. Recomendaciones del manejo diagnóstico-terapeútico inicial y multidisciplinario de la sepsis grave en los servicios de urgencias hospitalarios. Documento de consenso (SEMES-SEMICYUC). Med Intensiva. 2007;31:375-87.

6. Guidet B, Aegerter P, Gauzit R, Meshaka P, Dreyfuss D, CUB- Réa Study Group. Incidence and impact of organ dysfunctions associated with sepsis. Chest. 2005;127:942-51.

7. Castellanos-Ortega A, Suberviola B, García-Astudillo LA, Holanda MS, Ortiz F, Llorca J, et al. Impact of the Surviving Sepsis Campaign protocols on hospital length of stay and mortality in septic shock patients: results of a threeyear follow-up quasi experimental syudy. Crit Care Med. 2010;38:1036-43.

8. Marshall JC, Vincent JL, Fink MP, Cook DJ, Rubenfeld G, Foster D, et al. Measures, markers and mediators: toward a staging system for clinical sepsis. A report of the fifth Toronto sepsis roundtable. Toronto, Ontario, Canada. Crit Care Med. 2003;31:1560-7.

9. Marshall JC, Reinhart K. For the International Sepsis Forum. Biomarkers of sepsis. Crit Care Med. 2009;37:2290-8.

10. Ventetuolo CE, Levy MM. Biomarkers: diagnosis and risk assessment in sepsis. Clin Chest Med. 2008;29:591-603.

11. Gerlach $H$, Toussaint S. Sensitive, specific, predictive. statistical basics: how to use biomarkers. Crit Care Clin. 2011;27:215-27.

12. Tang BM, Eslick GD, Craig JC, McLean AS. Accuracy of procalcitonin for sepsis diagnosis in critically ill patients: systematic review and meta-analysis. Lancet Infect Dis. 2007;7:210-7.

13. Pierrakos C, Vincent JL. Sepsis biomarkers: a review. Crit Care. 2010;14(1):R15.

14. Liaudat S, Dayer E, Praz G, Bille J, Troillet N. Usefulness of procalcitonin serum level for the diagnosis of bacteremia. Eur J Clin Microbiol Infect Dis. 2001;20:524-7.

15. Zakariah AN, Cozzi SM, Van Nuffelen M, Clausi CM, Pradier O, Vincent JL. Combination of biphasic transmittance waveform with blood procalcitonin levels for diagnosis of sepsis in acutely ill patients. Crit Care Med. 2008;36:1507-12.

16. Deitcher SR, Eisenberg PR. Elevated concentrations of cross-linked fibrin degradation products in plasma. An early marker of gram-negative bacteremia. Chest. 1993;103:1107-12.

17. Schuetz P, Hausfater P, Amin D, Amin A, Haubitz S, Faessler L, et al. Biomarkers from distinct biological pathways improve early risk stratification in medical emergency patients: the multinational, prospective, observational TRIAGE study. Crit Care. 2015;19:377. 